\title{
COMMENTARY
}

\section{The need for standardized data reporting for prehospital airway management}

\author{
Daniel P Davis* \\ See related research by Lossius et al., http://ccforum.com/content/15/1/R26
}

\begin{abstract}
Despite a widespread belief in the value of aggressive prehospital airway management, the therapeutic benefits of early tracheal intubation (TI) remain unclear. In fact, most attempts to elucidate the benefits of prehospital $\mathrm{TI}$ on outcome from traumatic brain injury and cardiopulmonary arrest have documented an increase in mortality associated with the procedure. While some degree of selection bias is likely present in these studies, the inherent adverse physiological effects of intubation and a high incidence of desaturation and subsequent hyperventilation may indicate a harmful effect of the procedure. This uncertainty regarding such a fundamental resuscitation procedure as TI underscores the need for standardized data reporting in prehospital airway management research. To this end, the Utstein prehospital airway conference proposed a set of variables that would move us in that direction. However, the present article by Lossius and colleagues documents how far we still have to travel before such standardization can be achieved. Only through these efforts can we elucidate the true benefits - or harm - of advanced airway management during critical resuscitation.
\end{abstract}

First, let me applaud the authors for addressing a critical issue in the resuscitation of out-of-hospital patients [1]. Using axioms such as 'GCS 8, intubate' and the 'ABCs of resuscitation, we have assumed that aggressive airway management - including early tracheal intubation (TI) - is fundamental in the early management of critically ill and injured patients. Thus, it has been somewhat difficult to explain the growing body of literature associating early TI with increased mortality, even after adjustment for

${ }^{*}$ Correspondence: davismd@cox.net

Department of Emergency Medicine, UC San Diego, 200 West Arbor Drive, \#8676, San Diego, CA 92103-8676, USA multiple covariates reflecting the severity of injury or illness [2-5]. It is tempting to dismiss these studies as merely reflecting an inherent selection bias in that patients who can be intubated in the out-of-hospital environment - particularly without the use of neuromuscular blocking agents - have suffered a devastating and potentially fatal neurological injury. However, even controlled trials and analyses limiting enrollment to patients with intact airway reflexes requiring paralytics suggest a lack of benefit with early TI $[2,6]$.

On the other hand, there is a clear association between hypoxemia and mortality among brain-injured patients $[7,8]$. Although this is often reversible with non-invasive airway maneuvers, some patients may require a more aggressive approach to prevent hypoxic injury [9]. In addition, a recent analysis comparing actual outcomes to those predicted by the Trauma and Injury Severity Score (TRISS) suggests that early intubation is lifesaving among the most severely injured patients [10]. Furthermore, data from the Resuscitation Outcomes Consortium document decreased mortality among head-injured patients treated by emergency medical service (EMS) systems with higher intubation rates [11].

It is clear that suboptimal performance of intubation and ventilation plays an important role in outcomes. Elevated intracranial pressure, aspiration events, intubation failure, and post-intubation hyperventilation are associated with early TI and may impact survival [12-15]. With administration of neuromuscular blocking agents, desaturations during laryngoscopy can result in bradycardia and increased mortality [13,16]. For cardiac arrest victims, excessive ventilation rates and long compression pauses severely diminish cardiac output and adversely affect resuscitation success [17]. It remains unclear whether eliminating suboptimal intubation performance will allow the 'inherent benefits' of early intubation to emerge.

All of this suggests that the relationship between outof-hospital TI and outcome is complex and its therapeutic value remains uncertain. It is remarkable that this unproven and potentially harmful procedure remains firmly entrenched in consensus recommendations and protocols. 
One of the primary reasons for our uncertainty as to the benefit or harm of out-of-hospital TI is the difficulty in comparing reports from different EMS systems due to lack of uniform data reporting standards. Variability in patient populations, disease state, comorbidities, injury or illness severity, provider level-of-training and experience, intubation protocols, procedure performance, response/scene/transport intervals, use of neuromuscular blocking agents and sedatives, equipment, and disposition all likely affect outcomes but are inconsistently reported, as the present report demonstrates [1].

In addition, physiological criteria (hypoxemia, decreased level of consciousness, tachypnea or hypopnea) are used to justify early TI. However, few EMS systems routinely capture physiological data for subsequent review. These data have proven valuable in understanding the therapeutic impact of various procedures and may shed light on the relationship between TI and outcome [1]. For example, hypocapnia, whether obtained from out-ofhospital capnometry or arrival arterial blood gas, is independently associated with lower survival among head-injured patients $[18,19]$. Similarly, both hypoxemia and extreme hyperoxemia on trauma center arrival predict increased mortality [8].

The present study reveals that our objective of uniform data reporting for out-of-hospital TI remains elusive [1]. Some data elements were present in the majority of reports, while others were rarely included. This report may serve as stimulus to improve adherence for future studies. However, this would require that the proposed data elements be universally embraced, which may occur only after the importance of each is individually validated [20]. This may be challenging, since these elements cover a broad range of categories that may not be applicable in every investigation. For example, core patient variables may be relevant when considering intubation success rates, which would be unaffected by post-intubation variables. The proposed data set may undergo a period of refinement if particular elements are determined not to affect any of the outcome variables of interest. Finally, certain physiological variables may best be captured via export from monitors, which may not be feasible in some EMS systems.

Again, I would like to commend the authors in their efforts to document the variability with which proposed data elements are currently reported in out-of-hospital airway research [1]. This variability has prevented us from reaching conclusions regarding the optimal approach to airway management in early resuscitation due to the inability to appropriately account for covariates affecting patient outcomes, substantive differences in EMS system and provider characteristics, and intubation protocols. Only through uniform reporting can we follow the example set several decades ago by the original
Utstein conference participants in defining uniform reporting of data for cardiopulmonary arrest, which catalyzed important advances for our understanding of optimal therapy for these patients. I encourage investigators with interest in these areas to strongly consider adopting the recommended data elements so that we can begin to elucidate the optimal approach to out-of-hospital airway management.

\section{Abbreviations}

EMS, emergency medical service; TI, tracheal intubation.

\section{Competing interests}

The author declares that he has no competing interests.

Published: 14 March 2011

\section{References}

1. Lossius HM, Sollid SJM, Rehn M, Lockey DJ: Revisiting the value of prehospital tracheal intubation: an all time systematic literature review extracting the Utstein airway core variables. Crit Care 2011, 15:R26.

2. Davis DP, Hoyt DB, Ochs M, Fortlage D, Holbrook T, Marshall LK, Rosen P: The effect of paramedic rapid sequence intubation on outcome in patients with severe traumatic brain injury. J Trauma 2003, 54:444-453.

3. Davis DP, Peay J, Sise MJ, Vilke GM, Kennedy F, Eastman AB, Velky T, Hoyt DB: The impact of prehospital endotracheal intubation on outcome in moderate-to-severe traumatic brain injury. J Trauma 2005, 58:933-939.

4. Murray JA, Demetriades D, Berne TV, Stratton SJ, Cryer HG, Bongard F, Fleming A, Gaspard D: Prehospital intubation in patients with severe head injury. J Trauma 2000, 49:1065-1070.

5. Wang HE, Peitzman AD, Cassidy LD, Adelson PD, Yealy DM: Out-of-hospital endotracheal intubation and outcome after traumatic brain injury. Ann Emerg Med 2004, 44:439-450.

6. Gausche M, Lewis RJ, Stratton SJ, Haynes BE, Gunter CS, Goodrich SM, Poore PD, McCollough MD, Henderson DP, Pratt FD, Seidel JS: Effect of out-ofhospital pediatric endotracheal intubation on survival and neurological outcome: a controlled clinical trial. JAMA 2000, 283:783-790.

7. Chesnut RM, Marshall LF, Klauber MR, Blunt BA, Baldwin N, Eisenberg HM, Jane JA, Marmarou A, Foulkes MA: The role of secondary brain injury in determining outcome from severe head injury. J Trauma 1993, 34:216-222.

8. Davis DP, Meade W, Sise MJ, Kennedy F, Simon F, Tominaga G, Steele J, Coimbra R: Both hypoxemia and hyperoxemia may be detrimental in patients with severe traumatic brain injury. J Neurotrauma 2009, 26:2217-2223.

9. Davis DP, Fisher R, Buono C, Brainard C, Smith S, Ochs G, Poste JC, Dunford JV: Predictors of intubation success and therapeutic value of paramedic airway management in a large, urban EMS system. Prehosp Emerg Care 2006, 10:356-362.

10. Davis DP, Peay J, Sise MJ, Kennedy F, Simon F, Tominaga G, Steele J, Coimbra R: Prehospital airway and ventilation management: a trauma score and injury severity score-based analysis. J Trauma 2010, 69:294-301.

11. Davis DP, Koprowicz KM, Newgard CD, Daya M, Bulger EM, Stiell I, Nichol G, Stephens S, Dreyer J, Minei J, Kerby JD: The relationship between out-ofhospital airway management and outcome among trauma patients with Glasgow Coma Scale scores of 8 or less. Prehosp Emerg Care 2011 [Epub ahead of print].

12. Bozeman WP, Idris $A H$ : Intracranial pressure changes during rapid sequence intubation: a swine model. J Trauma 2005, 58:278-283.

13. Davis DP, Dunford J, Poste JC, Ochs M, Hoyt DB: The impact of hypoxia and hyperventilation on outcome following paramedic rapid sequence intubation of patients with severe traumatic brain injury. J Trauma 2004, 57:1-10.

14. Vadeboncoeur TF, Davis DP, Ochs M, Poste JC, Hoyt DB, Vilke GM: The ability of paramedics to predict aspiration in patients undergoing prehospital rapid sequence intubation. J Emerg Med 2006, 30:131-136.

15. Wang HE, Sweeney TA, O'Connor RE, Rubinstein H: Failed prehospital intubations: an analysis of emergency department courses and outcomes. Prehosp Emerg Care 2001, 5:134-141. 
16. Dunford JV, Davis DP, Ochs M, Doney M, Hoyt DB: Incidence of transient hypoxia and pulse rate reactivity during paramedic rapid sequence intubation. Ann Emerg Med 2003, 42:721-728.

17. Wang HE, Simeone SJ, Weaver MD, Callaway CW: Interruptions in cardiopulmonary resuscitation from paramedic endotracheal intubation. Ann Emerg Med 2009, 54:645-652.

18. Davis DP, Douglas DJ, Koenig W, Carrison D, Buono CJ, Dunford JV: Hyperventilation following aero-medical rapid sequence intubation may be a deliberate response to hypoxemia. Resuscitation 2007, 73:354-361.

19. Davis DP, Idris AH, Sise MJ, Kennedy F, Eastman AB, Velky T, Vilke GM, Hoyt DB:
Early ventilation and outcome in patients with moderate to severe traumatic brain injury. Crit Care Med 2006, 34:1202-1208.

20. Sollid SJM, Lockey D, Lossius HM, group PAAMe: A consensus-based template for uniform reporting of data from pre-hospital advanced airway management. Scand J Trauma Resusc Emerg Med 2009, 17:58.

doi:10.1186/cc10039

Cite this article as: Davis DP: The need for standardized data reporting for prehospital airway management. Critical Care 2011, 15:133. 